\title{
Allocating Multiple Estates among Agents with Single-Peaked Preferences*
}

\author{
Anirban $\operatorname{Kar}^{\dagger}$ \\ Özgür Kıbrıs ${ }^{\ddagger}$
}

July 1, 2006

\begin{abstract}
We consider the problem of allocating multiple social endowments (estates) of a perfectly divisible commodity among a group of agents with single-peaked preferences when each agent's share can come from at most one estate. We assume that an agent only cares about the size of her share and not the identity of the estate from which it comes. We inquire if well-known single-estate rules, such as the Uniform rule, the Proportional rule or the fixed-path rules can be coupled with a matching rule so as to achieve efficiency in the multi-estate level. We first observe that on the class of problems where all agents have symmetric preferences, any efficient single-estate rule can be extended to an efficient multi-estate rule. We however obtain negative results on the full preference domain.
\end{abstract}

${ }^{*}$ We would like to express our gratitude to Bhaskar Dutta, Semih Koray, Hervé Moulin, and Yuntong Wang for detailed comments. We also thank the seminar participants at Bilkent University, Indian Statistical Institute, Bilgi University, University of Warwick, ASSET 2003, and BWED XXVI. The usual disclaimer applies.

${ }^{\dagger}$ Department of Economics, University of Warwick. E-mail: A.Kar@warwick.ac.uk

${ }^{\ddagger}$ Corresponding author. Address: Faculty of Arts and Social Sciences, Sabanci University, Istanbul, Turkey. Phone: +90-216-483-9267, fax: +90-216-483-9250, e-mail: ozgur@sabanciuniv.edu 


\section{Introduction}

The problem of sharing a fixed amount of a perfectly divisible commodity has received a great deal of attention in the recent literature on mechanism design. This issue arises when members of a joint venture have to share costs or surpluses associated with the venture or when a scarce resource has to be allocated among competing users (for example, fishing rights in the North sea among European Union members or $\mathrm{SO}_{2}$ emission permits among coal burning power plants). ${ }^{1}$

In an influential paper, Sprumont (1991) analyzes this problem under a single-peaked preferences assumption. He proposes a uniform rationing rule (henceforth, the Uniform rule): when there is excess demand, the Uniform rule gives each agent the smaller of a common amount and the agent's most preferred consumption. Conversely, each agent receives the larger of these two amounts in case of excess supply.

On Sprumont's (1991) framework, many desirable properties are jointly compatible. To name a few, the Uniform rule satisfies Pareto efficiency, strategy proofness, anonymity, noenvy, equal division lower bound, peak-only, continuity, consistency, converse consistency, resource monotonicity, population monotonicity, and welfare domination under preference replacement (see Thomson (1996) for definitions and discussion of these properties). ${ }^{2}$ Based on these observations, several characterizations of the Uniform rule are presented (e.g. see Sprumont (1991), Ching (1992, 1994), and Thomson (1994a, 1994b)).

The literature also shows that very large classes of rules satisfy Pareto efficiency and strategy proofness. Barberà, Jackson, and Neme (1997) identify the class of allocation rules that satisfy Pareto efficiency, strategy proofness, and a monotonicity property. Moulin (1999)

\footnotetext{
${ }^{1}$ See Kibris (2003) for an axiomatic analysis of the problem of allocating freely disposable emission permits among firms each equipped with a single-peaked "profit function" and an exogenous constraint on its emissions.

${ }^{2}$ There are two alternative formulations of the last three properties. The first [second] type of formulation requires all agents' welfare [shares] be similarly affected by a change in the problems parameters. The Uniform rule satisfies the second type of formulations as well as weaker forms of the first type.
} 
and Ehlers (2002) show that Pareto efficiency, strategy proofness, resource monotonicity and consistency characterize a large class of fixed-path rules. This class includes both symmetric methods, such as the Uniform rule, and asymmetric methods like priority rules.

This abundance of positive results looks promising about the existence of "good" solutions to the following extension of this problem: there are multiple estates (each containing a social endowment of the commodity in question), the agents are first to be assigned to these estates and then the endowment of each estate is to be allocated among the agents assigned to it. An agent can be assigned to only one estate. ${ }^{3}$ We analyze this problem under the assumption that the agents only care about their consumption of the commodity and not the estate they are assigned to.

This problem seems to be a good abstraction of several real-life problems where a social endowment exists in several physically disjoint estates or where the agents have to be served in multiple isolated markets. ${ }^{4}$ Consider, for instance, a manager that has to assign workers to projects and in each project has to allocate the workload (e.g., the ministry of education hiring teachers for public schools, or a department head in a consulting company forming project teams). If the projects are at distant locations or if they require developing specialized knowledge, it is natural to require each worker to be assigned to only one project. Note that a worker with strictly convex preferences on money-leisure bundles has single-peaked preferences on the boundary of his budget set, and thus on his workload. ${ }^{5}$

\footnotetext{
${ }^{3}$ Without a constraint on the assignments, feasible allocations for our extended problem is no different than those for a single-estate problem which has a social endowment that is equal in amount to the sum of the existing estates' endowments.

${ }^{4}$ Indeed, rationing methods for markets in disequilibrium have been central in the construction of microeconomic foundations to Keynesian theories. Benassy (1982) presents a detailed discussion. He also discusses some problems that can arise when there are multiple isolated markets (e.g. see page 22).

${ }^{5}$ As another example, consider a university administration that has to allocate the capacity of two servers among departments. If there is a proportionally shared fixed cost of maintaining the servers, departmental preferences will typically be single-peaked because more time on the server increases the department's cost. Typically, each department prefers to be associated with only one server, although it is indifferent between the servers. In this case, an allocation problem has two components or stages. First, the university has to
} 
Gensemer, Hong, and Kelly $(1996,1998)$ analyze a similar environment where agents with single-peaked preferences are partitioned into several "islands" in each of which a given rule allocates the island's social endowment among its inhabitants. The authors introduce a concept of "migration equilibrium" which requires that no agent be better-off by migrating from their current island to another. They show that if the allocation rules used by the islands satisfy certain desirable properties, migration equilibria can fail to exist. ${ }^{6}$

While Gensemer et al $(1996,1998)$ question the "stability" implications of allocation rules, we question the efficiency implications. We suppose it is institutionally feasible to enforce a partition of the agents to the estates (e.g. consider the migration barriers used by countries). We then focus on the possibility of constructing efficient rules for the multiestate division problem. ${ }^{7}$ Such rules have two components: a matching rule that assigns the agents to the estates, and a (single-estate) allocation rule used in each estate. ${ }^{8}$

Our purpose is to understand if well-known single-estate rules, such as the Uniform rule, the Proportional rule or the fixed-path rules can be coupled with a matching rule so as to achieve efficiency in the multi-estate level. Our first result is encouraging to this end. In Proposition 3.1, we observe that on the class of problems where all agents have symmetric preferences, any efficient single-estate rule can be extended to an efficient multi-estate rule. Furthermore, we construct a matching rule whose combination with any efficient singleassign departments to servers, and then divide the total capacity of each server amongst the departments being serviced by the server.

${ }^{6}$ More specifically, the 1996 paper analyzes allocation rules that satisfy two of Pareto efficiency, strategy proofness, and no-envy. The 1998 paper analyzes the Proportional, Queueing, Uniform, and Egalitarian rules.

${ }^{7}$ An allocation is multi-estate (Pareto) efficient if no alternative way of partitioning the agents and allocating the estates makes an agent better-off without making any other worse-off. Unlike in single-estate problems where efficiency is equivalent to all agents' consuming at the same side of their peaks, an efficient multi-estate allocation can be such that there is excess supply in one estate and excess demand in the other (thus agents in different estates are consuming at the opposite sides of their peaks).

${ }^{8}$ Note that our problem is technically a hybrid of one-sided many-to-one matching and single-estate allocation problems. The issues involved thus resemble those in both problems. 
estate allocation rule produces multi-estate efficiency.

The positive conclusions of Proposition 3.1 do not hold on the full preference domain. Not all single-estate rules can be coupled with a matching rule so as to achieve multi-estate efficiency. In Theorem 3.1, we observe that all nondictatorial fixed-path rules suffer from this problem. ${ }^{9}$ The Uniform rule is also a fixed-path rule. Therefore, a simple corollary of our theorem establishes the impossibility of multi-estate efficiency if the Uniform rule is used at the single-estate level.

In Theorem 3.2, we analyze single-estate rules that satisfy an informational simplicity property called peak-only. This property requires a single-estate rule only to depend on the agents' most preferred consumption levels (that is, their peaks). In real-life applications, using more information than the agents' peaks is not practical. Thus, most of the well-known rules satisfy this property. ${ }^{10}$ The fixed-path rules as well as others, such as the Proportional rule, are all peak-only. We show that any peak-only single-estate rule that satisfies a very weak fairness condition (which requires agents with "extreme demands" not to be fully compensated) violates multi-estate efficiency.

In the above results, we impose all axioms (except efficiency) on the single-estate rules. This is logically weaker than imposing the multi-estate formulations of the same axioms on multi-estate rules. That is, if a multi-estate rule satisfies one of our axioms, then so does its

\footnotetext{
${ }^{9}$ We in fact prove this result for all single estate rules that satisfy efficiency, strategy proofness, consistency, and resource monotonicity. On a related domain, Moulin (1999) shows that these properties characterize the fixed-path rules. On our domain, all fixed-path rules satisfy these properties but there might be other rules that also do satisfy them.

${ }^{10}$ On a related note, the literature also contains models where a social endowment is to be allocated among a group of agents each equipped with a demand scalar (as opposed to a preference relation). For more, see Thomson (1995) and Moulin (2000).
} 
single-estate component. ${ }^{11}$ But the converse is not true. ${ }^{12}$ Therefore, theorems 3.1 and 3.2 also state the lack of multi-estate rules that satisfy the given axioms.

\section{Model}

There is a finite set $\mathcal{N}$ of potential agents. Assume $|\mathcal{N}| \geq 4$. A society is a finite subset $N$ of $\mathcal{N}$. The consumption space is denoted by $Z=[0,1]$. Each agent $i \in \mathcal{N}$ is equipped with a preference relation $R_{i}$ over $Z$. Let $P_{i}\left(I_{i}\right)$ be the strict preference (indifference) relation corresponding to $R_{i}$. A preference relation $R_{i}$ is single-peaked if there is $p\left(R_{i}\right) \in Z$, the peak of $R_{i}$, such that for all $x, y \in Z, x<y \leq p\left(R_{i}\right)$ or $x>y \geq p\left(R_{i}\right)$ implies $y P_{i} x$. Let $\mathcal{R}$ be the set of all single-peaked preference relations on $Z$. A single-peaked preference relation $R \in \mathcal{R}$ is symmetric if for each $x, y \in Z,|x-p(R)|=|y-p(R)|$ implies $x I y$. Let $\mathcal{R}_{\text {sym }}$ be the set of all symmetric preference relations on $Z$. Given $N \subset \mathcal{N}$, a preference profile $R_{N}$ is a list $\left(R_{i}\right)_{i \in N}$ such that for all $i \in N, R_{i} \in \mathcal{R}$. The set of all such profiles is $\mathcal{R}^{N}$. Given $R_{N} \in \mathcal{R}^{N}, p\left(R_{N}\right)=\left(p\left(R_{i}\right)\right)_{i \in N}$. For $\mathbf{x} \in \mathbb{R}^{N}, p\left(R_{N}\right)>\mathbf{x}$ means that for all $i \in N$, $p\left(R_{i}\right)>x_{i}$.

A single-estate economy for $N$ is a preference profile $R_{N} \in \mathcal{R}^{N}$ and an endowment scalar $\omega \in Z .{ }^{13}$ Let $\mathcal{E}_{1}^{N}$ denote the set of all single-estate economies $\left(R_{N}, \omega\right)$. Let $\mathcal{E}_{1}=\bigcup_{N \subset \mathcal{N}} \mathcal{E}_{1}^{N}$. A single-estate (allocation) rule is a function $f: \mathcal{E}_{1} \longrightarrow \bigcup_{N \subset \mathcal{N}} Z^{N}$ that satisfies $\sum_{i \in N} f_{i}\left(R_{N}, \omega\right)=\omega$ for all $\left(R_{N}, \omega\right) \in \mathcal{E}_{1}$. Note that the definition of $f$ allows the allocation method to depend on the coalition for which it is being used.

We will next introduce some basic properties for such rules. A single-estate rule $f$ is

\footnotetext{
${ }^{11}$ This is simply due to the existence of degenerate multi-estate problems where there is only one estate with a positive social endowment (please see Section 2). A multi-estate rule coincides with its single-estate component on this subdomain. Therefore, any property satisfied by a multi-estate rule on this subdomain passes on to its single-estate component.

${ }^{12}$ For example, consider a multi-estate rule which is a combination of the Uniform (single-estate) rule and a matching rule where the agents with symmetric preferences have dictatorial power. This multi-estate rule is manipulable though it is strategy-proof on the single estate level.

${ }^{13}$ The only difference between our domain and that of Sprumont (1991) is that he fixes $\omega=1$.
} 
(Pareto) efficient if for all $\left(R_{N}, \omega\right) \in \mathcal{E}_{1}$, there is no $\mathbf{x} \in Z^{N}$ with $\sum_{i \in N} x_{i}=\omega$ such that for all $i \in N, x_{i} R_{i} f_{i}\left(R_{N}, \omega\right)$ and for some $i \in N, x_{i} P_{i} f_{i}\left(R_{N}, \omega\right)$. For single-estate rules, Pareto efficiency is equivalent to the following property (Sprumont, 1991): for all $\left(R_{N}, \omega\right) \in \mathcal{E}_{1}$, $\omega \leq \sum_{N} p\left(R_{i}\right)\left[\omega \geq \sum_{N} p\left(R_{i}\right)\right]$ implies $f_{i}\left(R_{N}, \omega\right) \leq p\left(R_{i}\right)\left[f_{i}\left(R_{N}, \omega\right) \geq p\left(R_{i}\right)\right]$ for all $i \in N$.

Our next property rules out individual manipulations by requiring that no agent is betteroff by misrepresenting his preference information: a single-estate rule $f$ is strategy proof if for all $\left(R_{N}, \omega\right) \in \mathcal{E}_{1}, i \in N$, and $R_{i}^{\prime} \in \mathcal{R} ; f_{i}\left(R_{i}, R_{-i}, \omega\right) R_{i} f_{i}\left(R_{i}^{\prime}, R_{-i}, \omega\right)$.

Moulin (1999) introduces a new class of single-estate rules which can be described as follows. For each $N \subseteq \mathcal{N}$, an N-path, $g(N): Z \rightarrow Z^{N}$ satisfies $^{14}(a)$ for each $\lambda \in Z$, $\sum_{N} g_{i}(N, \lambda)=\lambda$ and for each $i \in N, g_{i}(N, \lambda) \leq \lambda$ and $(b)$ for each $\lambda, \lambda^{\prime} \in Z$ such that $\lambda \leq \lambda^{\prime}$ and for each $i \in N, g_{i}(N, \lambda) \leq g_{i}\left(N, \lambda^{\prime}\right)$. Let $\gamma(g(N))$ denote the range of $g(N)$. That is, $\gamma(g(N))=\{g(N, \lambda) \mid \lambda \in Z\}$. A full path $g=(g(N))_{N \subseteq \mathcal{N}}$ specifies for each $N \subseteq \mathcal{N}$, an $N$-path and satisfies $(c)$ for each $N \subseteq N^{\prime} \subseteq \mathcal{N}, \operatorname{proj}_{N}\left[\gamma\left(g\left(N^{\prime}\right)\right)\right]=\gamma(g(N))$. For each pair of full paths, $g^{+}$and $g^{-}$, the associated fixed-path rule $\phi^{\left(g^{+}, g^{-}\right)}$is defined as follows. For each $\left(R_{N}, \omega\right) \in \mathcal{E}_{1},(i)$ if $\omega \leq \sum_{N} p\left(R_{j}\right)$, there is $\lambda \in Z$ such that for each $i \in N$, $\phi_{i}^{\left(g^{+}, g^{-}\right)}\left(R_{N}, \omega\right)=\min \left\{p\left(R_{i}\right), g_{i}^{+}(N, \lambda)\right\}$ and $\sum_{N} \min \left\{p\left(R_{i}\right), g_{i}^{+}(N, \lambda)\right\}=\omega$; and $(i i)$ if $\omega>$ $\sum_{N} p\left(R_{j}\right)$, there is $\lambda \in Z$ such that for each $i \in N, \phi_{i}^{\left(g^{+}, g^{-}\right)}\left(R_{N}, \omega\right)=\max \left\{p\left(R_{i}\right), g_{i}^{-}(N, \lambda)\right\}$ and $\sum_{N} \max \left\{p\left(R_{i}\right), g_{i}^{-}(N, \lambda)\right\}=\omega$.

All fixed-path rules satisfy efficiency, strategy proofness as well as the following two properties. The first property requires that an increase in the social endowment never results in a decrease in someone's share: a single-estate rule $f$ is resource monotonic (Thomson, 1994a) if for each $\left(R_{N}, \omega\right),\left(R_{N}, \omega^{\prime}\right) \in \mathcal{E}_{1}$ such that $\omega<\omega^{\prime}$, we have $f_{i}\left(R_{N}, \omega\right) \leq f_{i}\left(R_{N}, \omega^{\prime}\right)$ for each $i \in N{ }^{15}$ The second property ensures that an agent $i$ leaving the economy with his assigned share does not affect the remaining agents' shares: a single-estate rule $f$ is consistent (Thomson, 1994b) if for all $N \subset \mathcal{N},\left(R_{N}, \omega\right) \in \mathcal{E}_{1}^{N}$, and $i \in N, f_{N \backslash\{i\}}\left(R_{N}, \omega\right)=$

\footnotetext{
${ }^{14}$ Abusing notation, for $\lambda \in Z$ we write $g(N, \lambda)$ instead of $g(N)(\lambda)$.

${ }^{15}$ More precisely, Thomson (1994a) discusses a version of this property which requires that, in response to a change in the social endowment, the "welfare" (as opposed to the share) of all agents be affected in the same direction.
} 
$f_{N \backslash\{i\}}\left(R_{N \backslash\{i\}}, \omega-f_{i}\left(R_{N}, \omega\right)\right)$.

The following property requires a rule only to use "simple" information about the agents: a single-estate rule $f$ is peak-only if for each $\left(R_{N}, \omega\right),\left(R_{N}^{\prime}, \omega\right) \in \mathcal{E}_{1}, p\left(R_{N}\right)=p\left(R_{N}^{\prime}\right)$ implies $f\left(R_{N}, \omega\right)=f\left(R_{N}^{\prime}, \omega\right)$. In real-life problems, it is much more practical to ask the agents to declare a most preferred amount rather than a whole preference relation. Thus most well-known rules, such as the Proportional rule or the fixed-path rules, are peak-only. These rules also satisfy the following property which requires that small perturbations in the data of a problem do not have a big effect on the agents' shares. Let $\lambda$ be the Lebesque measure on $Z$. The metric $\delta: \mathcal{R}^{2} \rightarrow \mathbb{R}_{+}$is defined as follows: for $R, R^{\prime} \in \mathcal{R}, \delta\left(R, R^{\prime}\right)=$ $\max _{x \in Z}\left|\lambda(\{y \in Z \mid y R x\})-\lambda\left(\left\{y \in Z \mid y R^{\prime} x\right\}\right)\right|$. Given $N \subset \mathcal{N}$, the metric $\delta_{N}: \mathcal{R}^{N} \times \mathcal{R}^{N} \rightarrow$ $\mathbb{R}_{+}$is defined as follows: for $R_{N}, R_{N}^{\prime} \in \mathcal{R}^{N}, \delta_{N}\left(R_{N}, R_{N}^{\prime}\right)=\max _{i \in N} \delta\left(R_{i}, R_{i}^{\prime}\right)$. A single-estate rule $f$ is continuous if for each $\left(R_{N}, \omega\right) \in \mathcal{E}_{1}$ and $\varepsilon>0$, there is $\zeta>0$ such that for each $R_{N}^{\prime} \in \mathcal{R}^{N}, \delta_{N}\left(R_{N}, R_{N}^{\prime}\right)<\zeta$ implies $\sum_{i \in N}\left(f_{i}\left(R_{N}, \omega\right)-f_{i}\left(R_{N}^{\prime}, \omega\right)\right)^{2}<\varepsilon$.

The following property rules out dictatoriality: a single-estate rule $f$ is nondictatorial if there is no $N \subset \mathcal{N}$ with $|N|>1, i \in N$, and $\omega \in Z$ such that either $(i)$ for all $R_{N} \in \mathcal{R}^{N}$ with $\sum_{N} p\left(R_{j}\right)>\omega, f_{i}\left(R_{N}, \omega\right)=\min \left\{\omega, p\left(R_{i}\right)\right\}$ (i.e. agent $i$ dictates when there is excess demand) or (ii) for all $R_{N} \in \mathcal{R}^{N}$ with $\sum_{N} p\left(R_{j}\right)<\omega, f_{i}\left(R_{N}, \omega\right)=p\left(R_{i}\right)$ (i.e. agent $i$ dictates when there is excess supply).

The focus of our paper is on economies where there are multiple estates of the same commodity. Let $S$ be the (finite) set of estates and assume $2 \leq|S| \leq|\mathcal{N}|$. An endowment vector is denoted by $\Omega=\left(\Omega_{1}, \ldots, \Omega_{|S|}\right) \in Z^{|S|}$. For $|N| \geq|S|$, a multi-estate economy for $N$ is a preference profile $R_{N} \in \mathcal{R}^{N}$ and an endowment vector $\Omega \in Z^{|S|} \backslash\{\mathbf{0}\}$. Let $\mathcal{E}^{N}$ denote the set of all multi-estate economies $\left(R_{N}, \Omega\right)$. Let $\mathcal{E}=\underset{\substack{N \subset \mathcal{N} \\|N| \geq|S|}}{\bigcup} \mathcal{E}^{N}$. Let $\mathcal{E}_{\text {sym }}$ be the subset of multi-estate problems where every agent has symmetric preferences. The set of all feasible allocations for $\left(R_{N}, \Omega\right) \in \mathcal{E}$ is

$$
X\left(R_{N}, \Omega\right)=\left\{x \in Z^{N} \mid \text { for some partition }\left[M_{1}, \ldots, M_{|S|}\right] \text { of } N, \quad \sum_{i \in M_{k}} x_{i}=\Omega_{k}, k \in S\right\} .
$$

A multi-estate rule $\Phi$ is a combination of $(i)$ a (matching) rule that assigns the agents 
to the estates and $(i i)$ a single-estate (allocation) rule that distributes each estate among the agents assigned to it. A matching rule $\mu$ associates each $N \subset \mathcal{N}$ and $\left(R_{N}, \Omega\right) \in \mathcal{E}^{N}$ with a partition of $N, \mu\left(R_{N}, \Omega\right)=\left[\mu_{1}\left(R_{N}, \Omega\right), \ldots, \mu_{|S|}\left(R_{N}, \Omega\right)\right]$ such that for each $k \in S, \mu_{k}\left(R_{N}, \Omega\right)=$ $\varnothing$ if and only if $\Omega_{k}=0 .{ }^{16} \mathrm{~A}$ multi-estate rule is a function $\Phi: \mathcal{E} \rightarrow \bigcup_{\left(R_{N}, \Omega\right) \in \mathcal{E}} X\left(R_{N}, \Omega\right)$ that, for a single-estate rule $f$ and a matching rule $\mu$, satisfies

$$
\Phi_{j}\left(R_{N}, \Omega\right)=f_{j}\left(R_{\mu_{k}\left(R_{N}, \Omega\right)}, \Omega_{k}\right) \text { if } j \in \mu_{k}\left(R_{N}, \Omega\right)
$$

for each $\left(R_{N}, \Omega\right) \in \mathcal{E}$ and $j \in N$. We denote this with $\Phi=(f, \mu)$.

Let $\mathcal{E}_{\text {deg }}$ be the set of degenerate multi-estate problems $\left(R_{N}, \Omega\right)$ where exactly one estate has a positive endowment, that is, there is $k \in S$ such that $\Omega_{k}>0$ and for all $l \in S \backslash\{k\}, \Omega_{l}=0$. By definition of a matching rule, the multi-estate rule $\Phi$ coincides with its single-estate component $f$ on the subdomain $\mathcal{E}_{\mathrm{deg}}$, that is, $\left.\Phi\right|_{\mathcal{E}_{\operatorname{deg}}}=f$.

We are primarily interested in efficiency. A multi-estate rule $\Phi$ is efficient if for all $N \subset \mathcal{N}$ and $\left(R_{N}, \Omega\right) \in \mathcal{E}^{N}$, there exists no $z \in X\left(R_{N}, \Omega\right)$ such that $z_{k} R_{k} \Phi_{k}\left(R_{N}, \Omega\right)$ for all $k \in N$ and $z_{l} P_{l} \Phi_{l}\left(R_{N}, \Omega\right)$ for some $l \in N$. It follows from the previous paragraph that every efficient multi-estate rule $\Phi=(f, \mu)$ induces an efficient single-estate rule $f$. For efficiency of a multi-estate rule, how it partitions the agents is very important: a partition $\left[N_{1}, \ldots, N_{|S|}\right]$ of $N$ is a critical partition if for any $k, l \in S$ satisfying $\sum_{N_{k}} p\left(R_{i}\right)>\omega_{k}$ and $\sum_{N_{l}} p\left(R_{i}\right)<\omega_{l}$, there is no $j \in N_{k}$ such that $\sum_{N_{k} \backslash\{j\}} p\left(R_{i}\right) \geq \omega_{k}$ and $\sum_{N_{l} \cup\{j\}} p\left(R_{i}\right) \leq \omega_{l}$. Note that any allocation that is not associated with a critical partition is bound to be Pareto dominated. This can be done by moving an agent (such as $j$ in the definition) from an excess demand estate to an excess supply estate (from estate $k$ to $l$ in the definition); this move increases what is left to the others in the excess demand estate and decreases what is left to the others in the excess supply estate.

\footnotetext{
${ }^{16}$ This condition restricts the matching rule not to assign agents to empty estates (and to assign at least one agent to each nonempty estate). However, it does not restrict the set of feasible allocations: if there were agents assigned to an empty estate, each would have received a zero share. Thus these agents can equivalently be assigned to a nonempty estate and get the same (zero) amount.
} 


\section{Results}

In this paper we ask the following simple question. Is it possible to achieve an efficient allocation of the estates in $S$ if we restrict ourselves to a class of well-behaved single-estate rules? In other words, we impose several desirable properties over $f$ and investigate whether a matching rule $\mu$ can be constructed to obtain an efficient multi-estate rule $\Phi=(f, \mu)$.

We first observe that if the agents have symmetric preferences, any efficient single-estate rule can be extended to an efficient multi-estate rule.

Proposition 3.1 On $\mathcal{E}_{\text {sym }}$, there is a matching rule $\mu$ such that for every efficient singleestate rule $f$, the multi-estate rule $\Phi=(f, \mu)$ is efficient.

Proof: Let the matching rule $\mu$ be defined as follows: for each $R_{N} \in \mathcal{R}_{s y m}^{N}, \mu\left(R_{N}, \Omega\right)$ is a partition of $N$ that minimizes

$$
\sum_{k \in S}\left|\Omega_{k}-\sum_{j \in \Pi_{k}} p\left(R_{j}\right)\right|
$$

over all partitions $\Pi=\left[\Pi_{1}, \ldots, \Pi_{|S|}\right]$ of $N$.

Let $f$ be any efficient single-estate allocation rule. We define a multi-estate rule $\Phi=$ $(f, \mu)$. Let $\left(R_{N}, \Omega\right) \in \mathcal{E}$ and let $x=\Phi\left(R_{N}, \Omega\right)$.

Note that, $\sum_{k \in S}\left|\Omega_{k}-\sum_{j \in \Pi_{k}} p\left(R_{j}\right)\right|=\sum_{k \in S}\left|\sum_{j \in \Pi_{k}}\left(x_{j}-p\left(R_{j}\right)\right)\right|$. For all $k$, due to efficiency of single-estate rules, the expression $\left(x_{j}-p\left(R_{j}\right)\right)$ has the same sign for all $j \in \Pi_{k}$. Hence

$$
\sum_{k \in S}\left|\Omega_{k}-\sum_{j \in \Pi_{k}} p\left(R_{j}\right)\right|=\sum_{i \in N}\left|x_{i}-p\left(R_{i}\right)\right| .
$$

Suppose $x$ is Pareto dominated by an allocation $y$. Then for all $i \in N,\left|y_{i}-p\left(R_{i}\right)\right| \leq$ $\left|x_{i}-p\left(R_{i}\right)\right|$, with strict inequality for at least one agent. Thus,

$$
\sum_{i \in N}\left|y_{i}-p\left(R_{i}\right)\right|<\sum_{i \in N}\left|x_{i}-p\left(R_{i}\right)\right|
$$

Without loss of generality we can assume that $y$ is efficient, corresponding to a partition $\Pi^{\prime}$. Then the same steps as above show that,

$$
\sum_{i \in N}\left|y_{i}-p\left(R_{i}\right)\right|=\sum_{k \in S}\left|\Omega_{k}-\sum_{j \in \Pi_{k}^{\prime}} p\left(R_{j}\right)\right| .
$$


This however, implies

$$
\sum_{k \in S}\left|\Omega_{k}-\sum_{j \in \Pi_{k}^{\prime}} p\left(R_{j}\right)\right|<\sum_{k \in S}\left|\Omega_{k}-\sum_{j \in \mu_{k}\left(R_{N}, \Omega\right)} p\left(R_{j}\right)\right|
$$

a contradiction.

In the proof of Proposition 3.1, we construct a matching rule that picks certain critical partitions. These partitions make use of the very particular structure of symmetric preferences (where an agent, independent of whether he receives more or less than his peak, becomes better-off as the distance between his peak and his share decreases).

Proposition 3.1 fails on the full domain of preferences. The following theorem implies that nondictatorial fixed-path rules can not be coupled with a matching rule so as to achieve multi-estate efficiency. All fixed-path rules satisfy efficiency, strategy proofness, resource monotonicity and consistency. ${ }^{17}$ We thus proceed to show that a nondictatorial singleestate rule that satisfies these properties can not be combined with a matching rule to satisfy multi-estate efficiency.

Theorem 3.1 For every single-estate rule $f$ that is efficient, nondictatorial, strategy proof, resource monotonic, and consistent, there is no matching rule $\mu$ such that $\Phi=(f, \mu)$ is efficient in multi-estate problems.

The proof makes use of the following lemmata. The first two, due to Ching (1994), are very useful in identifying the implications of two central properties, efficiency and strategy proofness, on single-estate rules. Next, we present them without proof.

Lemma 3.1 (Lemma 1 in Ching, 1994) Let $f$ satisfy efficiency and strategy proofness. Then for each $N \subset \mathcal{N}, i \in N$, and $\left(R_{i}, R_{-i}, \omega\right),\left(R_{i}^{\prime}, R_{-i}, \omega\right) \in \mathcal{E}_{1}^{N}$, if $p\left(R_{i}\right) \leq p\left(R_{i}^{\prime}\right)$, then $f_{i}\left(R_{i}, R_{-i}, \omega\right) \leq f_{i}\left(R_{i}^{\prime}, R_{-i}, \omega\right)$.

\footnotetext{
${ }^{17}$ On a slightly different domain, Moulin (1999) and Ehlers (2002) show that these properties characterize the class of fixed-path rules. On their domain, the consumption set of each agent $i$ is $\left[0, X_{i}\right]$ for some $X_{i} \in \mathbb{R}_{++}$(while we normalize all $X_{i}=1$ ) and $\omega \in\left[0, \sum_{N} X_{i}\right]$ (while we restrict $\omega \in[0,1]$ ). Due to these differences, their uniqueness proof does not directly apply to our domain (e.g., see Ehlers (2002), Lemma 3.2, where he uses the intermediate value theorem).
} 
It follows from this lemma that if $p\left(R_{i}\right)=p\left(R_{i}^{\prime}\right)$, then $f_{i}\left(R_{i}, R_{-i}, \omega\right)=f_{i}\left(R_{i}^{\prime}, R_{-i}, \omega\right)$.

Lemma 3.2 (Lemma 2 in Ching, 1994) Let $f$ satisfy efficiency and strategy proofness. Then for each $N \subset \mathcal{N}, i \in N$, and $\left(R_{i}, R_{-i}, \omega\right),\left(R_{i}^{\prime}, R_{-i}, \omega\right) \in \mathcal{E}_{1}^{N}$,

(i) if $p\left(R_{i}\right)<f_{i}\left(R_{i}, R_{-i}, \omega\right)$ and $p\left(R_{i}^{\prime}\right) \leq f_{i}\left(R_{i}, R_{-i}, \omega\right)$, then $f_{i}\left(R_{i}^{\prime}, R_{-i}, \omega\right)=f_{i}\left(R_{i}, R_{-i}, \omega\right)$,

(ii) if $p\left(R_{i}\right)>f_{i}\left(R_{i}, R_{-i}, \omega\right)$ and $p\left(R_{i}^{\prime}\right) \geq f_{i}\left(R_{i}, R_{-i}, \omega\right)$, then $f_{i}\left(R_{i}^{\prime}, R_{-i}, \omega\right)=f_{i}\left(R_{i}, R_{-i}, \omega\right)$.

The following two lemmata identify the implications of nondictatoriality on efficient and strategy proof rules. Please see the Appendix for their proofs. The first lemma states that in two-agent economies, for each agent there is a limit such that he is allotted his peak if and only if his peak is inside this limit.

Lemma 3.3 Let $f$ be an efficient, strategy proof, and nondictatorial single-estate rule. Let $N=\{1,2\}$. Then for each $\omega \in Z \backslash\{0\}$ and $i \in N$, there is $m_{i}^{d}(N, \omega) \in(0, \omega)$ and $m_{i}^{s}(N, \omega) \in(0, \omega)$ such that for any $R_{N} \in \mathcal{R}^{N}$,

$$
\text { if } p\left(R_{1}\right)+p\left(R_{2}\right)>\omega, \text { then }\left[p\left(R_{i}\right)>m_{i}^{d}(N, \omega) \Leftrightarrow f_{i}\left(R_{N}, \omega\right)<p\left(R_{i}\right)\right]
$$

and

$$
\text { if } p\left(R_{1}\right)+p\left(R_{2}\right)<\omega \text {, then }\left[p\left(R_{i}\right)<m_{i}^{s}(N, \omega) \Leftrightarrow f_{i}\left(R_{N}, \omega\right)>p\left(R_{i}\right)\right] \text {. }
$$

For more than two agents, the issue becomes much more complicated since fixing the share of an agent no longer determines the shares of the others. Nevertheless, a similar implication holds for rules that additionally satisfy continuity and consistency. The following lemma states that if we fix the preferences of all agents other than two, say $i$ and $j$, then in excess supply problems there is a limit for $i$ such that if his peak is under this limit (no matter what the preferences of $j$ are) $i$ can not be allotted his peak. Nondictatoriality of $f$ corresponds to this limit being in the interior of $Z$.

Lemma 3.4 Let $f$ be an efficient, strategy proof, consistent, continuous, and nondictatorial single-estate rule. Then for each $N \subset \mathcal{N}, \omega \in Z, i \in N, j \in N \backslash\{i\}$, and $R_{N \backslash\{i, j\}} \in \mathcal{R}^{N \backslash\{i, j\}}$ 
satisfying $p\left(R_{N \backslash\{i, j\}}\right)>0$, there is $m_{i}^{s}\left(\{i, j\}, R_{N \backslash\{i, j\}}, \omega\right) \in(0, \omega)$ such that for any $R_{i}, R_{j} \in \mathcal{R}$

$$
\text { if } \sum_{N} p\left(R_{k}\right)<\omega, \text { then }\left[p\left(R_{i}\right)<m_{i}^{s}\left(\{i, j\}, R_{N \backslash\{i, j\}}, \omega\right) \Rightarrow f_{i}\left(R_{N}, \omega\right)>p\left(R_{i}\right)\right] \text {. }
$$

Note that the observation of Lemma 3.4 holds for all nondictatorial fixed-path rules. We next prove the theorem.

Proof of Theorem 3.1: Assume that $f$ is efficient, nondictatorial, strategy proof, resource monotonic, and consistent. Then,$f$ is also continuous. ${ }^{18}$ As a result, lemmas 3.3 and 3.4 apply to $f$. We prove the theorem in two steps. In Step 1, we construct an economy with two estates and agents $|N| \geq 4$. In Step 2, we extend this economy to another with an arbitrary number of (say $m$ ) estates and agents $|N| \geq m+2$.

Step 1: We construct a two estate problem for which any allocation that $\Phi$ can choose is Pareto dominated. First, let $\Omega=(\omega, \omega)$ where $\omega>0$. Let $n \geq 4, N=\{1,2, \ldots, n\}$, $N^{\prime}=N \backslash\{n\}$, and $\hat{N}=N^{\prime} \backslash\{1,2,3\}$. Define

$$
D=\left\{p \in Z^{N^{\prime}} \mid \sum_{N^{\prime}} p_{k}>\omega \text { and for all } l \in N^{\prime}, \sum_{N^{\prime} \backslash\{l\}} p_{k}<\omega\right\} \text {. }
$$

Note that for all $p \in D$ and $l \in N^{\prime}, p_{l}>0$. Let $R_{N^{\prime}} \in \mathcal{R}^{N^{\prime}}$ be such that $p\left(R_{N^{\prime}}\right) \in D$.

We first claim that for each $k \in N^{\prime}, f_{k}\left(R_{N^{\prime}}, \omega\right)>0$. For this, note that by efficiency, for each $k \in N^{\prime}, f_{k}\left(R_{N^{\prime}}, \omega\right) \leq p\left(R_{k}\right)$. Suppose there is $l \in N^{\prime}$ such that $f_{l}\left(R_{N^{\prime}}, \omega\right)=0$. Then $\sum_{N^{\prime}} f_{k}\left(R_{N^{\prime}}, \omega\right)=\sum_{N^{\prime} \backslash\{l\}} f_{k}\left(R_{N^{\prime}}, \omega\right) \leq \sum_{N^{\prime} \backslash\{l\}} p\left(R_{k}\right)<\omega$, a contradiction to the definition of a single estate rule.

Then by efficiency of $f$ and by the definition of $D$, there is $i \in N^{\prime}$ such that

$$
0<f_{i}\left(R_{N^{\prime}}, \omega\right)<p\left(R_{i}\right)
$$

Without loss of generality assume that $i=1$.

\footnotetext{
${ }^{18}$ Since $f$ is efficient and strategy proof, a similar argument to Lemma 1 in Sprumont (1991) establishes that $f_{i}\left(R_{i}, R_{N \backslash i}, \omega\right)$ is continuous in $R_{i}$. Also, by resource monotonicity, $f_{j}\left(R_{i}, R_{N \backslash i}, \omega\right)$ is continuous in $\omega$. Since $\left(\omega-f_{i}\left(R_{i}, R_{N \backslash i}, \omega\right)\right)$ is continuous in $R_{i}, f_{j}\left(R_{N \backslash i}, \omega-f_{i}\left(R_{i}, R_{N \backslash i}, \omega\right)\right)$ is also continuous in $R_{i}$. Thus by consistency, $f_{j}\left(R_{i}, R_{N \backslash i}, \omega\right)$ is continuous in $R_{i}$.
} 
We next construct a preference profile $R_{N}^{*} \in \mathcal{R}^{N}$ that satisfies two lists of properties: $A 1$ to $A 5$ and $B 1$ to $B 4$. Inequality (1), Lemma 3.3, and Lemma 3.4 ensure that, for each inequality stated in properties $A 1$ to $A 5$, the right hand side is strictly between 0 and $\omega$ (and thus, the inequality is well-defined).

$A 1$. For each $k \in \hat{N}$, let $R_{k}^{*}$ be such that $0<p\left(R_{k}^{*}\right)<\min \left\{m_{k}^{d}(\{k, n\}, \omega), f_{k}\left(R_{N^{\prime}}, \omega\right)\right\}$. By Lemma 3.3, $p\left(R_{k}^{*}\right)<m_{k}^{d}(\{k, n\}, \omega)$ implies that agent $k$ gets his peak. Thus $A 1$ ensures that if only two agents, $k$ and $n$, are assigned to one of the estates and if they happen to be in excess demand, then agent $k$ gets his peak.

$A 2$. Let $R_{3}^{*}$ be such that $0<p\left(R_{3}^{*}\right)<\min \left\{m_{3}^{s}\left(\{1,3\}, R_{\hat{N}}^{*}, \omega\right), m_{3}^{d}(\{3, n\}, \omega), f_{3}\left(R_{N^{\prime}}, \omega\right)\right\}$. Consider a situation where the coalition $\hat{N} \cup\{1,3\}$ has been assigned to one of the estates and the preference profile for $\hat{N}$ is $R_{\hat{N}}^{*}$. If this coalition happens to be in excess supply, then by Lemma $3.4, p\left(R_{3}^{*}\right)<m_{3}^{s}\left(\{1,3\}, R_{\hat{N}}^{*}, \omega\right)$ guarantees that agent 3 does not get his peak. Similarly if only two agents, $k$ and $n$, are assigned to an estate and if they happen to be in excess demand, then by Lemma 3.3, $p\left(R_{3}^{*}\right)<m_{3}^{d}(\{3, n\}, \omega)$ guarantees that agent 3 will get his peak.

$A 3$. Let $R_{2}^{*}$ be such that

$$
0<p\left(R_{2}^{*}\right)<\min \left\{\begin{array}{c}
\min _{k \in N^{\prime} \backslash\{1,2\}}\left\{m_{2}^{s}\left(\{1,2\}, R_{N^{\prime} \backslash\{1,2, k\}}^{*}, \omega\right), p\left(R_{k}^{*}\right)\right\}, \\
m_{2}^{s}\left(\{2,3\}, R_{\hat{N}}^{*}, \omega\right), m_{2}^{d}(\{2, n\}, \omega), f_{2}\left(R_{N^{\prime}}, \omega\right), p\left(R_{1}\right)
\end{array}\right\} .
$$

Take any $k \in N^{\prime} \backslash\{1,2\}$. Consider a situation where the coalition $N^{\prime} \backslash\{k\}$ has been assigned to one of the estates and the preference profile for $N^{\prime} \backslash\{1,2, k\}$ is $R_{N^{\prime} \backslash\{1,2, k\}}^{*}$. If this coalition happens to be in excess supply, then by Lemma 3.4, $p\left(R_{2}^{*}\right)<m_{2}^{s}\left(\{1,2\}, R_{N^{\prime} \backslash\{1,2, k\}}^{*}, \omega\right)$ guarantees that agent 2 does not get his peak. Moreover, $p\left(R_{2}^{*}\right)<p\left(R_{k}^{*}\right)$ implies that agent 2 has the smallest peak of $\{2, \ldots, n-1\}$. By Lemma 3.4, $p\left(R_{2}^{*}\right)<m_{2}^{s}\left(\{2,3\}, R_{\hat{N}}^{*}, \omega\right)$ guarantees that if the coalition $N^{\prime} \backslash\{1\}$ is assigned to an estate, if the preference profile of $\hat{N}$ is $R_{\hat{N}}^{*}$, and if this coalition is in excess supply then agent 2 does not get his peak. Similarly by Lemma 
3.3, $p\left(R_{2}^{*}\right)<m_{2}^{d}(\{2, n\}, \omega)$ guarantees that agent 2 will get his peak when only two agents, 2 and $n$, are sharing an estate and they are in excess demand.

$A 4$. Let $R_{1}^{*}$ be such that $p\left(R_{1}^{*}\right) \geq p\left(R_{1}\right)$ and $p\left(R_{N^{\prime}}^{*}\right) \in D .^{19}$

Note that $p\left(R_{N^{\prime}}^{*}\right) \in D$ implies that for all $k \in N^{\prime}$, the coalition $N^{\prime} \backslash k$ is in excess supply and hence, we can later apply $A 2$ and $A 3$.

A5. Let $R_{n}^{*}$ be such that $\omega>p\left(R_{n}^{*}\right)>\max \left\{m_{n}^{d}(\{1, n\}, \omega), \max _{j \in N^{\prime}}\left\{\omega-p\left(R_{j}^{*}\right)\right\}\right\}$.

By Lemma 3.3, $p\left(R_{n}^{*}\right)>m_{n}^{d}(\{1, n\}, \omega)$ guarantees that agent $n$ will not get his peak when only two agents, 1 and $n$, are sharing an estate and they are in excess demand. The inequality $p\left(R_{n}^{*}\right)>\max _{j \in N^{\prime}}\left\{\omega-p\left(R_{j}^{*}\right)\right\}$ implies that agent $n$ will be in an excess demand situation with each agent $j \in N^{\prime}$ and by $A 1, A 2$, and $A 3$, will not get his peak.

Claim. $f_{1}\left(R_{N^{\prime}}^{*}, \omega\right)<p\left(R_{1}^{*}\right)$ and for each $i \in N^{\prime} \backslash\{1\}, f_{i}\left(R_{N^{\prime}}^{*}, \omega\right)=p\left(R_{i}^{*}\right)$.

\section{Proof of Claim.}

First note that $f_{1}\left(R_{N^{\prime}}, \omega\right)<p\left(R_{1}\right) \leq p\left(R_{1}^{*}\right)$ (by Inequality (1) and $A 4$, respectively). At the profile $\left(R_{1}^{*}, R_{N^{\prime} \backslash 1}\right)$, the coalition $N^{\prime}$ is at excess demand. Thus, by Lemma 3.2, $f_{1}\left(R_{\{1\}}^{*}, R_{\{2, \ldots, n-1\}}, \omega\right)=f_{1}\left(R_{\{1, \ldots, n-1\}}, \omega\right)<p\left(R_{1}^{*}\right)$. By consistency

$$
f\left(R_{\{1\}}^{*}, R_{N^{\prime} \backslash 1}, \omega\right)=f\left(R_{N^{\prime}}, \omega\right)
$$

For each $i \in N^{\prime} \backslash\{1\}$, let $R_{i}^{\prime}$ be such that $p\left(R_{i}^{\prime}\right)=f_{i}\left(R_{\{1\}}^{*}, R_{\{2, \ldots, n-1\}}, \omega\right)$. Thus $p\left(R_{i}^{\prime}\right) \leq p\left(R_{i}\right)$.

Now consider agent $i$ changing his preference relation to $R_{i}^{\prime}$ at profile $\left(R_{1}^{*}, R_{\{2, \ldots, i-1\}}^{\prime}, R_{\{i, \ldots, n-1\}}\right)$.

Both of these profiles create excess demand since

$$
p\left(R_{1}^{*}\right)+\sum_{j=2}^{i} p\left(R_{j}^{\prime}\right)+\sum_{j=i+1}^{n-1} p\left(R_{j}\right)=p\left(R_{1}^{*}\right)+\sum_{j=2}^{i} f_{j}\left(R_{\{1\}}^{*}, R_{\{2, \ldots, n-1\}}, \omega\right)+\sum_{j=i+1}^{n-1} p\left(R_{j}\right)>\omega .
$$

By Lemma 3.2, $f_{i}\left(R_{1}^{*}, R_{\{2, \ldots, i-1\}}^{\prime}, R_{\{i, \ldots, n-1\}}, \omega\right)=f_{i}\left(R_{1}^{*}, R_{\{2, \ldots, i\}}^{\prime}, R_{\{i+1, \ldots, n-1\}}, \omega\right)$. Then by consistency $f_{N^{\prime}}\left(R_{1}^{*}, R_{\{2, \ldots, i-1\}}^{\prime}, R_{\{i, \ldots, n-1\}}, \omega\right)=f_{N^{\prime}}\left(R_{1}^{*}, R_{\{2, \ldots, i\}}^{\prime}, R_{\{i+1, \ldots, n-1\}}, \omega\right)$. Iteratively

\footnotetext{
${ }^{19}$ Note that this is possible since for all $k \in N^{\prime} \backslash\{1\}, p\left(R_{k}^{*}\right)<f_{k}\left(R_{N^{\prime}}, \omega\right) \leq p\left(R_{k}\right)$.
} 
applying this argument to agents $2,3, \ldots,(n-1)$ gives us

$$
f\left(R_{\{1\}}^{*}, R_{\{2, \ldots, n-1\}}^{\prime}, \omega\right)=f\left(R_{\{1\}}^{*}, R_{\{2, \ldots, n-1\}}, \omega\right) .
$$

Therefore, $f_{1}\left(R_{\{1\}}^{*}, R_{\{2, \ldots, n-1\}}^{\prime}, \omega\right)<p\left(R_{1}^{*}\right)$ and

$$
f_{N^{\prime} \backslash\{1\}}\left(R_{\{1\}}^{*}, R_{\{2, \ldots, n-1\}}^{\prime}, \omega\right)=\left(p\left(R_{2}^{\prime}\right), \ldots, p\left(R_{n-1}^{\prime}\right)\right) .
$$

We next show that $f_{N^{\prime} \backslash\{1\}}\left(R_{\{1,2\}}^{*}, R_{\{3, \ldots, n-1\}}^{\prime}, \omega\right)=\left(p\left(R_{2}^{*}\right), p\left(R_{3}^{\prime}\right), \ldots, p\left(R_{n-1}^{\prime}\right)\right)$. By properties $A 1, A 2, A 3$, and Equation (2), for all $i \in N^{\prime} \backslash\{1\}$,

$$
p\left(R_{i}^{*}\right)<f_{i}\left(R_{N^{\prime}}, \omega\right)=f_{i}\left(R_{\{1\}}^{*}, R_{N^{\prime} \backslash 1}, \omega\right)=p\left(R_{i}^{\prime}\right) .
$$

This and $\sum_{N^{\prime}} p\left(R_{i}^{*}\right)>\omega$ implies, by $A 4$, that

$$
p\left(R_{1}^{*}\right)+p\left(R_{2}^{*}\right)+\sum_{N^{\prime}} p\left(R_{i}^{\prime}\right)>\omega
$$

Then by efficiency, $f_{2}\left(R_{\{1,2\}}^{*}, R_{\{3, \ldots, n-1\}}^{\prime}, \omega\right) \leq p\left(R_{2}^{*}\right)$. Suppose, $f_{2}\left(R_{\{1,2\}}^{*}, R_{\{3, \ldots, n-1\}}^{\prime}, \omega\right)<$ $p\left(R_{2}^{*}\right)$. Let $R_{2}^{* *} \in \mathcal{R}$ be such that $p\left(R_{2}^{* *}\right)=p\left(R_{2}^{*}\right)$ and $p\left(R_{2}^{\prime}\right) P_{2}^{* *} f_{2}\left(R_{\{1,2\}}^{*}, R_{\{3, \ldots, n-1\}}^{\prime}, \omega\right)$. Then by Lemma 3.1

$$
f_{2}\left(R_{1}^{*}, R_{2}^{* *}, R_{\{3, \ldots, n-1\}}^{\prime}, \omega\right)=f_{2}\left(R_{\{1,2\}}^{*}, R_{\{3, \ldots, n-1\}}^{\prime}, \omega\right)
$$

implies $p\left(R_{2}^{\prime}\right) P_{2}^{* *} f_{2}\left(R_{1}^{*}, R_{2}^{* *}, R_{\{3, \ldots, n-1\}}^{\prime}, \omega\right)$. This contradicts strategy proofness. Therefore,

$$
f_{2}\left(R_{\{1,2\}}^{*}, R_{\{3, \ldots, n-1\}}^{\prime}, \omega\right)=p\left(R_{2}^{*}\right)
$$

Now by consistency,

$$
f_{N^{\prime} \backslash\{2\}}\left(R_{\{1\}}^{*}, R_{\{2, \ldots, n-1\}}^{\prime}, \omega\right)=f_{N^{\prime} \backslash\{2\}}\left(R_{\{1\}}^{*}, R_{\{3, \ldots, n-1\}}^{\prime}, \omega-p\left(R_{2}^{\prime}\right)\right)
$$

and

$$
f_{N^{\prime} \backslash\{2\}}\left(R_{\{1,2\}}^{*}, R_{\{3, \ldots, n-1\}}^{\prime}, \omega\right)=f_{N^{\prime} \backslash\{2\}}\left(R_{\{1\}}^{*}, R_{\{3, \ldots, n-1\}}^{\prime}, \omega-p\left(R_{2}^{*}\right)\right) .
$$

Note that $\omega-p\left(R_{2}^{*}\right)>\omega-p\left(R_{2}^{\prime}\right)$. Thus, by resource monotonicity

$$
f_{N^{\prime} \backslash\{2\}}\left(R_{\{1\}}^{*}, R_{\{3, \ldots, n-1\}}^{\prime}, \omega-p\left(R_{2}^{\prime}\right)\right) \leq f_{N^{\prime} \backslash\{2\}}\left(R_{\{1\}}^{*}, R_{\{3, \ldots, n-1\}}^{\prime}, \omega-p\left(R_{2}^{*}\right)\right)
$$


Now,

$$
\begin{aligned}
f_{N^{\prime} \backslash\{1,2\}}\left(R_{\{1,2\}}^{*}, R_{\{3, \ldots, n-1\}}^{\prime}, \omega\right) & =f_{N^{\prime} \backslash\{1,2\}}\left(R_{\{1\}}^{*}, R_{\{3, \ldots, n-1\}}^{\prime}, \omega-p\left(R_{2}^{*}\right)\right) & & \text { (by (7)) } \\
& \geq f_{N^{\prime} \backslash\{1,2\}}\left(R_{\{1\}}^{*}, R_{\{3, \ldots, n-1\}}^{\prime}, \omega-p\left(R_{2}^{\prime}\right)\right) & & \text { (by (8)) } \\
& =f_{N^{\prime} \backslash\{1,2\}}\left(R_{\{1\}}^{*}, R_{\{2, \ldots, n-1\}}^{\prime}, \omega\right) & & \text { (by (6)) } \\
& \geq\left(p\left(R_{3}^{\prime}\right), \ldots, p\left(R_{n-1}^{\prime}\right)\right) & & \text { (by (3)). }
\end{aligned}
$$

But, from (4), we know that the profile $\left(R_{\{1,2\}}^{*}, R_{\{3, \ldots, n-1\}}^{\prime}\right)$ is in excess demand and hence by efficiency

$$
f_{N^{\prime} \backslash\{1,2\}}\left(R_{\{1,2\}}^{*}, R_{\{3, \ldots, n-1\}}^{\prime}, \omega\right)=\left(p\left(R_{3}^{\prime}\right), \ldots, p\left(R_{n-1}^{\prime}\right)\right) .
$$

Then, by equalities (5) and (9), $f_{N^{\prime} \backslash\{1\}}\left(R_{\{1,2\}}^{*}, R_{\{3, \ldots, n-1\}}^{\prime}, \omega\right)=\left(p\left(R_{2}^{*}\right), p\left(R_{3}^{\prime}\right), \ldots, p\left(R_{n-1}^{\prime}\right)\right)$.

An iterative application of this argument for $i \in\{3, \ldots, n-1\}$, thus gives

$$
f_{N^{\prime} \backslash\{1\}}\left(R_{\{1, \ldots, n-1\}}^{*}, \omega\right)=\left(p\left(R_{2}^{*}\right), \ldots, p\left(R_{n-1}^{*}\right)\right)
$$

Since by $A 4, \sum_{N^{\prime}} p\left(R_{j}^{*}\right)>\omega$, feasibility implies $f_{1}\left(R_{N^{\prime}}^{*}, \omega\right)<p\left(R_{1}^{*}\right)$.

Other properties of the agents' preferences are as follows. Due to Lemma 3.1, changing an agent's preferences without changing his peak does not affect his share assigned by a single-estate rule. Furthermore, due to consistency, such a change does not affect the shares of others as well. Instead of creating a new preference profile with the same peaks as $R^{*}$ and stating each time that, as above, the agents' shares remain unchanged, we will abuse notation and state these properties directly on $R^{*}$. After each property, we state why it is compatible with those in $A 1$ to $A 5$.

B1. If $f_{1}\left(R_{\{1, n\}}^{*}, \omega\right)=p\left(R_{1}^{*}\right)$, let $\left(\omega-\sum_{\hat{N} \cup\{3\}} p\left(R_{k}^{*}\right)\right) P_{1}^{*} f_{1}\left(R_{N^{\prime}}^{*}, \omega\right)$. Otherwise, let $(\omega-$ $\left.\sum_{\hat{N} \cup\{3\}} p\left(R_{k}^{*}\right)\right) P_{1}^{*} \max \left\{f_{1}\left(R_{N^{\prime}}^{*}, \omega\right), f_{1}\left(R_{\{1, n\}}^{*}, \omega\right)\right\}$.

By $A 4, \omega-\sum_{\hat{N} \cup\{3\}} p\left(R_{k}^{*}\right)>p\left(R_{1}^{*}\right)$. By the Claim, $p\left(R_{1}^{*}\right)>f_{1}\left(R_{N^{\prime}}^{*}, \omega\right)$. Thus, $B 1$ does not contradict the previous properties.

B2. $\min \left\{\omega-\sum_{N^{\prime} \backslash\{2\}} p\left(R_{k}^{*}\right), \omega-p\left(R_{n}^{*}\right)\right\} P_{2}^{*} \min _{k \in N^{\prime} \backslash\{2\}}\left\{f_{2}\left(R_{N^{\prime} \backslash\{k\}}^{*}, \omega\right)\right\}$. 
By $A 4$ and $A 5, \min \left\{\omega-\sum_{N^{\prime} \backslash\{2\}} p\left(R_{k}^{*}\right), \omega-p\left(R_{n}^{*}\right)\right\}<p\left(R_{2}^{*}\right)$. Since by $A 3 p\left(R_{2}^{*}\right)<$ $\min _{k \in N^{\prime} \backslash\{1,2\}}\left\{m_{2}^{s}\left(\{1,2\}, R_{N^{\prime} \backslash\{1,2, k\}}^{*}, \omega\right), p\left(R_{k}^{*}\right)\right\}$, we have $p\left(R_{2}^{*}\right)<\min _{k \in N^{\prime} \backslash\{2\}}\left\{f_{2}\left(R_{N^{\prime} \backslash\{k\}}^{*}, \omega\right)\right\}$. B3. $\left(\omega-\sum_{N^{\prime} \backslash\{3\}} p\left(R_{k}^{*}\right)\right) P_{3}^{*} f_{3}\left(R_{N^{\prime} \backslash\{2\}}^{*}, \omega\right)$.

By $A 4, \omega-\sum_{k \in N^{\prime} \backslash\{3\}} p\left(R_{k}^{*}\right)<p\left(R_{3}^{*}\right)$ and by $A 2, p\left(R_{3}^{*}\right)<f_{3}\left(R_{N^{\prime} \backslash\{2\}}^{*}, \omega\right)$. B4. $\left(\omega-p\left(R_{2}^{*}\right)\right) I_{n}^{*} \omega$.

By $A 5, \omega-p\left(R_{2}^{*}\right)<p\left(R_{n}^{*}\right)<\omega$.

Note that by $A 3$ and $A 4, p\left(R_{2}^{*}\right)<p\left(R_{1}^{*}\right)$. Also, by $A 3, p\left(R_{2}^{*}\right)<p\left(R_{k}^{*}\right)$ for all $k \in N^{\prime} \backslash\{1\}$. Thus for all $k \in N^{\prime}, \omega-p\left(R_{2}^{*}\right)>\omega-p\left(R_{k}^{*}\right)$. Therefore, an important implication of $B 4$ is that $\omega P_{n}^{*}\left(\omega-p\left(R_{k}^{*}\right)\right)$ for all $k \in N^{\prime}$.

Now we will show that allocations under every possible partition can be Pareto dominated. Since $\Omega_{1}=\Omega_{2}=\omega$, partitions $\left[N_{1}, N_{2}\right]$ and $\left[N_{2}, N_{1}\right]$ are the same. The critical partitions are as follows:

$$
\begin{aligned}
& \text { 1. }\left[N^{\prime} \backslash\{k\},\{k, n\}\right] \text { for all } k \in \hat{N} \cup\{3\} \text {, } \\
& \text { 2. }\left[N^{\prime} \backslash\{2\},\{2, n\}\right] \text {, } \\
& \text { 3. }\left[N^{\prime} \backslash\{1\},\{1, n\}\right] \text {, } \\
& \text { 4. }\left[N^{\prime},\{n\}\right] \text {. }
\end{aligned}
$$

Now consider any partition of type 1 and the following allocation $z$ which is consistent with partition $\left[N^{\prime},\{n\}\right]$ :

$$
z_{k}= \begin{cases}p\left(R_{k}^{*}\right) & \text { if } k \neq 2, n, \\ \omega-\sum_{N^{\prime} \backslash\{2\}} p\left(R_{k}^{*}\right) & \text { if } k=2 \\ \omega & \text { if } k=n .\end{cases}
$$

Now for each $k \in N \backslash\{1,2, n\}$, by $A 1, f_{n}\left(R_{\{k, n\}}^{*}, \omega\right)=\omega-p\left(R_{k}^{*}\right)$ and by $B 4$, he is better-off at $z$. Also note that agent 2 , by $B 2$, is better-off at $z$. Finally, in $z$ every agent $k \in N \backslash\{2, n\}$ receives his peak. Therefore, $z$ is a Pareto improvement. 
Now consider Partition 2 and the following alternative allocation $y$ which is consistent with $\left[N^{\prime},\{n\}\right]$ :

$$
y_{k}= \begin{cases}p\left(R_{k}^{*}\right) & \text { if } k \neq 3, n, \\ \omega-\sum_{N^{\prime} \backslash\{3\}} p\left(R_{k}^{*}\right) & \text { if } k=3, \\ \omega & \text { if } k=n .\end{cases}
$$

By $A 3, f_{n}\left(R_{\{2, n\}}^{*}, \omega\right)=\omega-p\left(R_{2}^{*}\right)$ and by $B 4, n$ is indifferent. By $B 3$, agent 3 is strictly better-off. Finally, the rest are getting their peaks at $y$. So $y$ is a Pareto improvement.

There are two possible scenarios under Partition 3. If $f_{1}\left(R_{\{1, n\}}^{*}, \omega\right)=p\left(R_{1}^{*}\right)$ then as before, $z$ Pareto dominates this allocation. Alternatively if $f_{1}\left(R_{\{1, n\}}^{*}, \omega\right)<p\left(R_{1}^{*}\right)$, consider the following allocation $x$ which is consistent with $\left[N^{\prime} \backslash\{2\},\{2, n\}\right]$ :

$$
x_{k}= \begin{cases}p\left(R_{k}^{*}\right) & \text { if } k \neq 1,2, \\ \omega-\sum_{N^{\prime} \backslash\{1,2\}} p\left(R_{k}^{*}\right) & \text { if } k=1, \\ \omega-p\left(R_{n}^{*}\right) & \text { if } k=2 .\end{cases}
$$

Now by $B 1$, agent 1 is strictly better-off and by $B 2$, agent 2 is strictly better-off. All the other agents are getting their peaks at $x$. So $x$ is a Pareto improvement.

Finally consider Partition 4 and the following allocation $v$ which is consistent with the partition $\left[N^{\prime} \backslash\{2\},\{2, n\}\right]$ :

$$
v_{k}= \begin{cases}p\left(R_{k}^{*}\right) & \text { if } k \neq 1, n, \\ \omega-\sum_{N^{\prime} \backslash\{1,2\}} p\left(R_{k}^{*}\right) & \text { if } k=1, \\ \omega-p\left(R_{2}^{*}\right) & \text { if } k=n .\end{cases}
$$

By $B 4$, agent $n$ is indifferent. By $B 1$, agent 1 is strictly better-off. Finally, all the other agents are getting their peaks at $v$. Thus $v$ is a Pareto improvement.

Step 2: Here we extend the economy of Step 1 to another with an arbitrary number of estates. Let $m>2$ be the number of estates and let $N$ be the set of agents, where $|N| \geq m+2$. The following were the critical partitions in the two estate problem. 


$$
\begin{aligned}
& \text { 1. }\left[N^{\prime} \backslash\{k\},\{k, n\}\right] \text { for all } k \in \hat{N} \cup\{3\} \text {, } \\
& \text { 2. }\left[N^{\prime} \backslash\{2\},\{2, n\}\right] \text {, } \\
& \text { 3. }\left[N^{\prime} \backslash\{1\},\{1, n\}\right], \\
& \text { 4. }\left[N^{\prime},\{n\}\right] .
\end{aligned}
$$

We know that for all $k \in N^{\prime}$, the $N^{\prime} \backslash\{k\}$ coalitions are in excess supply and the $\{k, n\}$ coalitions are in excess demand. Similarly $N^{\prime}$ is in excess demand and $\{n\}$ is in excess supply. As $\left[N^{\prime},\{n\}\right]$ is a critical partition, $\sum_{N^{\prime}} p\left(R_{k}^{*}\right)>\omega$ and for all $l \in N^{\prime}, \sum_{N^{\prime} \backslash\{l\}} p\left(R_{k}^{*}\right)<\omega$. Now, in each excess demand situation, let us calculate the residual amount an agent will have to consume if all others receive their peaks. So for example in partition 2 , the residuals are $\left(\omega-p\left(R_{n}^{*}\right)\right)$ and $\left(\omega-p\left(R_{2}^{*}\right)\right)$. Let $\delta$ be the minimum of all such numbers and $p\left(R_{n}^{*}\right)$, that is,

$$
\delta=\min \left\{\min _{l \in N^{\prime}}\left\{\omega-p\left(R_{l}^{*}\right), \omega-\sum_{N^{\prime} \backslash\{l\}} p\left(R_{k}^{*}\right)\right\}, \omega-p\left(R_{n}^{*}\right), p\left(R_{n}^{*}\right)\right\} .
$$

Note that $\delta>0$. Assume that there is a $0<\theta<\min _{l \in N} p\left(R_{l}^{*}\right)$ such that $\omega P_{k}^{*} \theta$ for all $k \in N$. This does not contradict with any of the previous properties.

Now pick any number of estates $m \leq \min \{|\mathcal{N}|-2,|S|\}$ and extend the economy of Step 1 by adding $(m-2)$ new estates and $(m-2)$ new agents. These new estates are all of the same size $\varpi \in Z$, where $0<\varpi<\min \{\theta, \delta\}$. New agents, denoted by $l \in M=$ $\{(n+1), \ldots,(n+m-2)\}$ have identical preferences $R^{*} \in \mathcal{R}$, where $p\left(R^{*}\right)=\varpi$. We next show that in any efficient partition, each of the new agents will be assigned to one new estate. Suppose this is not true. Then one new estate must have been assigned to some agent $j \in N$ and one new agent $l \in M$ is part of a coalition $K \subset N \cup M \backslash\{j\}$ that occupies one of the two original estates. (More than one agent in any new estate is an inefficient partition, because such partition is not critical). We next create an alternative multi-estate allocation $x \in Z^{N \cup M}$ as follows. We swap agents $j$ and $l$ and we keep all other agents in the same estate as before. After the swap, we give agent $l$ his peak, $x_{l}=p\left(R^{*}\right)$. We also give the new coalition members of $j$ their peaks, $x_{k}=p\left(R_{k}^{*}\right)$ for all $k \in K \backslash\{l\}$, and we give agent $j$ the residual, $x_{j}=\omega-\sum_{k \in K \backslash\{l\}} x_{k}$. Finally, we give all other agents the same share as before. 
Note that, for all agents other than $j$, the new allocation is weakly preferred to the old one. We will next show that agent $j$ strictly prefers his new share $x_{j}$ to his old one $\varpi$. There are two possibilities.

Case 1) $\sum_{k \in K \cup\{j\}} p\left(R_{k}^{*}\right) \geq \omega$ (that is, agent $j$ ends up in an excess demand coalition after the swap). Then since $\varpi<\delta<x_{j} \leq p\left(R_{j}^{*}\right)$, we have $x_{j} P_{j}^{*} \varpi$.

Case 2) $\sum_{k \in K \cup\{j\}} p\left(R_{k}^{*}\right)<\omega$ (that is, agent $j$ ends up in an excess supply coalition after the swap). Then $p\left(R_{j}^{*}\right)<x_{j}<\omega$ implies $x_{j} P_{j}^{*} \omega$. Combining this with $\omega P_{j}^{*} \theta$ and $\theta>\varpi$, we obtain $x_{j} P_{j}^{*} \varpi$.

This argument establishes that in any efficient multi-estate allocation, each new estate must contain exactly one new agent. Thus, all agents in $N$ must be assigned to the first two estates (of Step 1). We are then back to Step 1 where we know an efficient multi-estate allocation does not exist.

Since the Uniform rule is a nondictatorial fixed-path rule, a straightforward corollary of Theorem 3.1 is that any multi-estate rule which coincides with the Uniform rule on the single-estate level is bound to be inefficient.

There are dictatorial single-estate rules that satisfy all other properties of Theorem 3.1 and that can be extended to an efficient multi-estate rule. The simplest example is a priority single-estate rule (with respect to an exogenous priority ordering of the agents) where the agent with $k^{\text {th }}$ priority picks his most preferred allocations among those picked by the agent with $(k-1)^{t h}$ priority. An efficient multi-estate extension of this rule is where, given the single-estate priority allocations in each estate, the agent with $k^{\text {th }}$ priority picks his most preferred matchings among those picked by the agent with $(k-1)^{t h}$ priority. This example can be manipulated to construct a nondictatorial rule that satisfies all properties of Theorem 3.1 except strategy proofness and that can be extended to an efficient multi-estate rule. For this, let the priority ranking be endogenously determined by ordering the agents with respect to the magnitude of their peaks (so the agent with the highest peak gets the first priority and so on) and by using an exogenous ordering to break the ties in peaks. 
Next, we analyze the implications of peak-only together with a weak fairness property. This property demands that those who are most responsible for the excess (demand or supply) must not be compensated fully. That is, in case of excess supply those who demanded the least, and in case of excess demand those who demanded the most must not be allocated their peaks: a single-estate rule $f$ satisfies nonaccommodation of extremes if for all $\left(R_{N}, \omega\right) \in \mathcal{E}_{1}$ and $i \in N$,

(i) $\sum_{N} p\left(R_{k}\right)<\omega$ and $p\left(R_{i}\right) \leq p\left(R_{j}\right)$ for all $j \in N$ implies $f_{i}\left(R_{N}, \omega\right)>p\left(R_{i}\right)$, and

(ii) $\sum_{N} p\left(R_{k}\right)>\omega$ and $p\left(R_{i}\right) \geq p\left(R_{j}\right)$ for all $j \in N$ implies $f_{i}\left(R_{N}, \omega\right)<p\left(R_{i}\right)$.

This property is satisfied by well-known rules such as the Uniform rule and the Proportional rule.

Theorem 3.2 For every single-estate rule $f$ that satisfies efficiency, peak-only and nonaccommodation of extremes, there is no matching rule $\mu$ such that $\Phi=(f, \mu)$ is efficient in multi-estate problems.

Proof: As in Theorem 3.1, the proof of this result is in two steps. First, we construct an economy with two estates and with agents $|N| \geq 4$ and second, we extend it to an arbitrary number of estates. Since the second step is similar to that of Theorem 3.1, it is omitted for brevity. We next implement the first step and construct the two-estate economy.

Suppose $f$ satisfies the given properties. Let $N=\{0,1, \ldots n\}$ and $N^{\prime}=\{1,2, \ldots, n\}$. Let $\Omega=(\omega, \omega)$ where $\omega>0$. Let $\eta \in \mathbb{R}$ satisfy $\frac{\omega}{n(n-1)}>\eta>0$. Let $R=\left(R_{0}, R_{1}, \ldots, R_{n}\right)$ be such that $p\left(R_{0}\right)=\frac{\omega(n-1)}{n}$ and for all $i \in N^{\prime}, p\left(R_{i}\right)=\frac{\omega}{n}+\eta$. Thus $p\left(R_{0}\right)>p\left(R_{i}\right)$ for all $i \in N^{\prime}$.

It is easy to check that the critical partitions are as follows:

1. $\left[\{0, i\}, N^{\prime} \backslash\{i\}\right]$ for $i \in N^{\prime}$,

2. $\left[\{0\}, N^{\prime}\right]$.

At partitions of type $1,\{0, i\}$ faces excess demand while $N^{\prime} \backslash\{i\}$ faces excess supply. At Partition 2, $N^{\prime}$ faces excess demand and $\{0\}$ faces excess supply. 
For each $i \in N^{\prime}$, let $\left(x_{0}^{i}, x_{i}^{i}\right)=f\left(R_{\{0, i\}}, \omega\right),\left(x_{j}^{i}\right)_{j \in N^{\prime} \backslash\{i\}}=f\left(R_{N^{\prime} \backslash\{i\}}, \omega\right)$. Also let $\left(x_{j}^{0}\right)_{j \in N^{\prime}}=$ $f\left(R_{N^{\prime}}, \omega\right)$. By efficiency and nonaccommodation of extremes, for all $i \in N^{\prime}, x_{0}^{i}<p\left(R_{0}\right)$ and for all $j \in N^{\prime} \backslash\{i\}$,

$$
x_{j}^{i}>p\left(R_{j}\right)
$$

Let $x_{0}=\max _{i \in N^{\prime}}\left\{x_{0}^{i}\right\}$ and $x_{n}=\min _{i \in N^{\prime} \backslash\{n\}}\left\{x_{n}^{i}\right\}$. We assume that the agents' preferences satisfy the following properties. Note that as single-estate rules are peak-only, these assumptions do not have any effect on the agents' shares.

1. $x_{0} I_{0} \omega$.

Note that by nonaccommodation of extremes, $x_{0}<p\left(R_{0}\right)$. Also, $p\left(R_{0}\right)<\omega$.

2. $\left(\frac{\omega}{n}-(n-1) \eta\right) P_{n} x_{n}$.

By inequality $(10), x_{n}>p\left(R_{n}\right)$ and by definition of $\eta, p\left(R_{n}\right)>\left(\frac{\omega}{n}-(n-1) \eta\right)$.

3. $\left(\frac{\omega}{n}-(n-1) \eta\right) P_{1} x_{1}^{n}$.

By inequality $(10), x_{1}^{n}>p\left(R_{1}\right)$ and by definition of $\eta, p\left(R_{1}\right)>\left(\frac{\omega}{n}-(n-1) \eta\right)$.

4. $\left(\frac{2 \omega}{n}-(n-2) \eta\right) P_{2} x_{2}^{0}$.

By nonaccommodation of extremes, $x_{2}^{0}<p\left(R_{2}\right)$ and by definition of $\eta, p\left(R_{2}\right)<\left(\frac{2 \omega}{n}-\right.$ $(n-2) \eta)$.

Now any partition of type 1 except $\left[\{0, n\}, N^{\prime} \backslash\{n\}\right]$ can be Pareto dominated by an allocation $x$, consistent with $\left[\{0\}, N^{\prime}\right]$ :

$$
x_{k}= \begin{cases}\frac{\omega}{n}+\eta & \text { if } k \neq 0, n, \\ \frac{\omega}{n}-(n-1) \eta & \text { if } k=n, \\ \omega & \text { if } k=0 .\end{cases}
$$

Note that in $x$, all $k \in N^{\prime} \backslash\{n\}$ receives his peak, agent $n$ is receiving a share that by Property 2 , he prefers to any $x_{n}^{i}$, and finally agent 0 receives $\omega$ which by Property 1 he weakly prefers to all $x_{0}^{i}$. 
The allocation corresponding to partition $\left[\{0, n\}, N^{\prime} \backslash\{n\}\right]$ is Pareto dominated by the following $y$ which is consistent again with Partition $\left[\{0\}, N^{\prime}\right]$ :

$$
y_{k}= \begin{cases}\frac{\omega}{n}+\eta & \text { if } k \neq 0,1 \\ \frac{\omega}{n}-(n-1) \eta & \text { if } k=1 \\ \omega & \text { if } k=0\end{cases}
$$

At allocation $y$, all agents but 0 and 1 get their peaks. agent 1 is better-off due to Property 3 . Finally, agent 0 is strictly better-off due to Property 1 . Therefore, $y$ is a Pareto improvement.

Now we analyze Partition 2. By feasibility, there is $l \in N^{\prime}$ such that $x_{l}^{0} \leq \frac{\omega}{n}$. This allocation can be Pareto dominated by the following $z$ which is consistent with Partition $\left[\{0, l\}, N^{\prime} \backslash\{l\}\right]:$

$$
z_{k}= \begin{cases}\frac{\omega}{n}+\eta & \text { if } k \neq 0,2, l, \\ \frac{2 \omega}{n}-(n-2) \eta & \text { if } k=2, \\ \omega-x_{0} & \text { if } k=l \\ x_{0} & \text { if } k=0 .\end{cases}
$$

By efficiency $x_{i}^{i}=\omega-x_{0}^{i} \leq p\left(R_{i}\right)$, which implies $\omega-x_{0} \leq \omega-x_{0}^{i} \leq p\left(R_{i}\right)$ for all $i \in N^{\prime}$. Particularly, $\omega-x_{0} \leq p\left(R_{l}\right)$. Now by nonaccommodation of extremes, $x_{0}<p\left(R_{0}\right)$. Thus, $p\left(R_{l}\right) \geq \omega-x_{0}>\omega-p\left(R_{0}\right)=\frac{\omega}{n}>x_{l}^{0}$. Therefore, $\left(\omega-x_{0}\right) P_{l} x_{l}^{0}$. By Property 4 , agent 2 is strictly better-off and by Property 1 , agent 0 is indifferent. Finally all the remaining agents get their peaks at $z$ and thus they are all better-off. This completes the proof.

There are peak-only single-estate rules that violate nonaccommodation of extremes and that can be extended to an efficient multi-estate rule. The simplest example is a priority rule (with respect to a fixed priority ordering).

\section{Concluding comments}

We assume that the agents do not have preferences on the estates. Without this assumption, the problem gets much richer and much more complicated. One of the simpler alternative 
cases is when the agents have lexicographic preferences (e.g. say a professor ranking job offers first looks at the name of a university and then at the teaching load). Under this assumption, our problem gets much "closer" to matching models. The analysis of this extension should present interesting open questions.

Finally, we focus on multi-estate rules that are "compositions" of a single-estate rule and a matching rule. These rules all satisfy an "estate neutrality" condition (which requires that the name of the estate does not affect the allocation method) and a "consistency" condition (which requires that if in two different problems the same set of agents is assigned to the same estate, those agents' shares should also be the same). ${ }^{20} \mathrm{~A}$ formal analysis of rules that violate these properties is left for future research. Nevertheless, we finish with an informal discussion of estate neutrality.

Multi-estate rules that violate estate neutrality use different single-estate rules in different estates. Thus, the number of allocations achievable via alternative matchings is much greater than that of an estate neutral multi-estate rule and (very informally) the existence of a preference profile where all resulting allocations are Pareto dominated becomes less likely. In the following example, we present a two-estate problem where using the Uniform rule in both estates leads to inefficiency, independent of which matching rule is chosen. In the same example however, using the Uniform rule in the first estate and a priority rule in the second one does not lead to a violation of efficiency.

Example 4.1: Let $p(R)=(0.4,0.4,0.55,0.8)$ and let $\Omega=(1,1)$. If the Uniform rule is

\footnotetext{
${ }^{20}$ If a multi-estate rule is not consistent in this sense, the way an estate is allocated can depend on the preferences of agents in other estates. For example, consider a multi-estate rule which uses a constant matching rule and a priority allocation rule where the priority ordering depends on agent 1's preferences (e.g. $(1, \ldots, n)$ if $0 R_{1} 1$ and $(n, \ldots, 1)$ if $\left.1 P_{1} 0\right)$. Now, as agent 1 's preferences change, the matching of the agents to the estates will remain the same but the allocation in each estate will change.
} 
used in both estates, the following allocations are attainable via critical partitions.

$\begin{array}{ccccc}\text { Partition } & x_{1} & x_{2} & x_{3} & x_{4} \\ {[\{1,2,3\},\{4\}]} & 0.33 & 0.33 & 0.33 & 1 \\ {[\{1,2\},\{3,4\}]} & 0.5 & 0.5 & 0.5 & 0.5 \\ {[\{1,3\},\{2,4\}]} & 0.45 & 0.5 & 0.55 & 0.5 \\ {[\{2,3\},\{1,4\}]} & 0.5 & 0.45 & 0.55 & 0.5\end{array}$

Each is Pareto dominated by the allocation

$$
x^{*}=(0.45,0.45,0.55,0.55)
$$

if $0.45 R_{i} 0.33$ for $i=1,2$ and $0.55 R_{4} 1$ with at least one preference being strict.

On the other hand, if the Uniform rule is used in the first estate and the Serial Dictatorship with priority order $(4,3,2,1)$ is used in the second, the allocations resulting from critical partitions are as follows.

$$
\begin{array}{ccccc}
\text { Partition } & x_{1} & x_{2} & x_{3} & x_{4} \\
{[\{1,2,3\},\{4\}]} & 0.33 & 0.33 & 0.33 & 1 \\
{[\{1,2\},\{3,4\}]} & 0.5 & 0.5 & 0.2 & 0.8 \\
{[\{1,3\},\{2,4\}]} & 0.45 & 0.2 & 0.55 & 0.8 \\
{[\{2,3\},\{1,4\}]} & 0.2 & 0.45 & 0.55 & 0.8 \\
{[\{4\},\{1,2,3\}]} & 0.05 & 0.4 & 0.55 & 1 \\
{[\{3,4\},\{1,2\}]} & 0.6 & 0.4 & 0.5 & 0.5 \\
{[\{2,4\},\{1,3\}]} & 0.45 & 0.4 & 0.55 & 0.6 \\
{[\{1,4\},\{2,3\}]} & 0.4 & 0.45 & 0.55 & 0.6
\end{array}
$$

The allocations $x^{1}=(0.45,0.2,0.55,0.8)$ and $x^{2}=(0.2,0.45,0.55,0.8)$ can not both be Pareto dominated, independent of how the agents' preferences are. In both, agents 3 and 4 receive their peaks. Since in any Pareto dominating allocation they should continue to do so, they should be in different estates and together with at least one other agent. Thus $x^{1}$ can only be Pareto dominated by $x^{2}$ or vice versa. But then the Pareto dominating allocation is efficient. 


\section{References}

[1] Barberà, S., M. Jackson and A. Neme (1997). Strategy-Proof Allotment Rules; Games and Economic Behavior 18;1-21.

[2] Benassy, J.P. (1982). The Economics of Market Disequilibrium. Academic Press.

[3] Ching, S. (1992). A Simple Characterization of the Uniform Rule; Economic Letters 40; 57-60.

[4] Ching, S. (1994). An Alternative Characterization of the Uniform Rule; Social Choice and Welfare $11 ; 131-136$.

[5] Ehlers, L. (2002). On Fixed-Path Rationing Methods; Journal of Economic Theory 106; $472-477$.

[6] Gensemer, S. H., Hong, L., Kelly, J. S. (1996). Division rules and migration equilibria; Journal of Economic Theory 69; 104-116.

[7] Gensemer, S. H., Hong, L., Kelly, J. S. (1998). Migration equilibrium and specific division rules; Social Choice and Welfare 15; 201-209.

[8] Kıbrıs, Ö. (2003). Constrained Allocation Problems with Single-Peaked Preferences: An Axiomatic Analysis, Social Choice and Welfare 20; 353-362.

[9] Moulin, H. (1980). On Strategy-Proofness and Single-Peakedness; Public Choice 35; 437-455.

[10] Moulin, H. (1999). Rationing a Commodity along Fixed Paths; Journal of Economic Theory 84; 41-72.

[11] Moulin, H. (2000). Priority Rules and Other Asymmetric Rationing Methods; Econometrica $68 ; 643-684$. 
[12] Sprumont, Y. (1991). The Division Problem with Single-Peaked Preferences: A Characterization of the Uniform Allocation Rule; Econometrica 59; 509-519.

[13] Thomson, W. (1994a). Resource Monotonic Solutions to the Problem of Fair Division when the Preferences are Single-Peaked; Social Choice and Welfare 11; 205-223.

[14] Thomson, W. (1994b). Consistent Solutions to the Problem of Fair Division when the Preferences are Single-Peaked; Journal of Economic Theory 63; 219-245.

[15] Thomson, W. (1995). Axiomatic Analysis of Bankruptcy and Taxation Problems: a Survey; mimeo., University of Rochester.

[16] Thomson, W. (1996). The Theory of Fair Allocation (unpublished manuscript).

\section{Appendix}

When there are two agents, an efficient and strategy proof single-estate rule can be described fully in terms of a median voter rule (Moulin 1980, Barberà et. al. 1997). A median voter rule is defined as follows. First, given that there are only two agents, preferences of agent 2 can be described in terms of agent 1's share. Essentially this is a one-dimensional choice problem where both agents have preference over the same object (which is agent 1's share). Then, median voter rules are of the following form; let $0 \leq p_{1} \leq p_{2} \leq \omega$, then:

$$
f_{1}\left(R_{1}, R_{2}, \omega\right)= \begin{cases}p\left(R_{1}\right) & \text { if } p_{1} \leq p\left(R_{1}\right) \leq p_{2}, \\ \text { median }\left\{p\left(R_{1}\right), \omega-p\left(R_{2}\right), p_{1}\right\} & \text { if } p\left(R_{1}\right)<p_{1}, \\ \text { median }\left\{p\left(R_{1}\right), \omega-p\left(R_{2}\right), p_{2}\right\} & \text { if } p\left(R_{1}\right)>p_{2} .\end{cases}
$$

Among such rules, nondictatorial ones can be characterized in Lemma 3.3.

Proof of Lemma 3.3: We prove this result for agent 1; the proof for agent 2 is exactly the same. If $p\left(R_{1}\right)+p\left(R_{2}\right)>\omega$, then by Equation $(11), f_{1}\left(R_{N}, \omega\right)=p\left(R_{1}\right)$ if and only if $p\left(R_{1}\right) \leq p_{2}$. Hence $m_{1}^{d}(N, \omega)=p_{2}$. Alternatively if $p\left(R_{1}\right)+p\left(R_{2}\right)<\omega, f_{1}\left(R_{N}, \omega\right)=p\left(R_{1}\right)$ if and only if $p\left(R_{1}\right) \geq p_{1}$. Therefore $m_{1}^{s}(N, \omega)=p_{1}$. Thus efficiency and strategy proofness 
are sufficient to imply the existence of such $m_{i}^{d}$ and $m_{i}^{s}$. Now, since $f$ is nondictatorial, $p_{1}$, $p_{2}$ and hence $m_{i}^{s}, m_{i}^{d}$ are strictly between 0 and $\omega$.

Proof of Lemma 3.4: The proof is by induction. For $|N|=2$, the conclusion follows from Lemma 3.3. Now, let $n \geq 3$ and assume that the conclusion holds for all $N \subset \mathcal{N}$ with $|N|<n$.

For contradiction, suppose there is $N^{0} \subset \mathcal{N}$ with $\left|N^{0}\right|=n, \omega^{0} \in Z, i, j \in N^{0}, R_{N^{0} \backslash\{i, j\}}^{0} \in$ $\mathcal{R}^{N^{0} \backslash\{i, j\}}$ with $p\left(R_{N^{0} \backslash\{i, j\}}^{0}\right)>0$, and for all $\varepsilon \in\left(0, \omega^{0}\right)$, there is $R_{i}^{\varepsilon}, R_{j}^{\varepsilon} \in \mathcal{R}$ such that $p\left(R_{i}^{\varepsilon}\right)<\varepsilon$ and $p\left(R_{i}^{\varepsilon}\right)+p\left(R_{j}^{\varepsilon}\right)+\sum_{N^{0} \backslash\{i, j\}} p\left(R_{l}^{0}\right)<\omega^{0}$, but $f_{i}\left(R_{i}^{\varepsilon}, R_{j}^{\varepsilon}, R_{N^{0} \backslash\{i, j\}}^{0}, \omega^{0}\right)=p\left(R_{i}^{\varepsilon}\right)$. Let $R_{N^{0}}^{\varepsilon}=\left(R_{i}^{\varepsilon}, R_{j}^{\varepsilon}, R_{N^{0} \backslash\{i, j\}}^{0}\right)$.

For each $k \in N^{0} \backslash\{i, j\}$, by the induction hypothesis applied to $N^{0} \backslash\{k\}$,

$$
m_{i}^{s}\left(\{i, j\}, R_{N^{0} \backslash\{i, j, k\}}^{0}, \omega^{0}-p\left(R_{k}^{0}\right)\right) \in\left(0, \omega^{0}\right) .
$$

Claim. For all $k \in N^{0} \backslash\{i, j\}, \varepsilon \in \mathbb{R}_{+}$with $0<\varepsilon<m_{i}^{s}\left(\{i, j\}, R_{N^{0} \backslash\{i, j, k\}}^{0}, \omega^{0}-p\left(R_{k}^{0}\right)\right)$, and $R_{i}^{\varepsilon}, R_{j}^{\varepsilon} \in \mathcal{R}$ as defined above, we have $f_{k}\left(R_{N^{0}}^{\varepsilon}, \omega^{0}\right)>p\left(R_{k}^{0}\right)$.

Proof of Claim. Since $p\left(R_{i}^{\varepsilon}\right)<m_{i}^{s}\left(\{i, j\}, R_{N^{0} \backslash\{i, j, k\}}^{0}, \omega^{0}-p\left(R_{k}^{0}\right)\right)$ and $p\left(R_{i}^{\varepsilon}\right)+p\left(R_{j}^{\varepsilon}\right)+$ $\sum_{N^{0} \backslash\{i, j\}} p\left(R_{l}^{0}\right)<\omega^{0}$, by the induction hypothesis applied to $N^{0} \backslash\{k\}$ we have

$$
f_{i}\left(R_{N^{0} \backslash\{k\}}^{\varepsilon}, \omega^{0}-p\left(R_{k}^{0}\right)\right)>p\left(R_{i}^{\varepsilon}\right)
$$

Now suppose $f_{k}\left(R_{N^{0}}^{\varepsilon}, \omega^{0}\right)=p\left(R_{k}^{0}\right)$. Then by consistency,

$$
f_{i}\left(R_{N^{0}}^{\varepsilon}, \omega^{0}\right)=f_{i}\left(R_{N^{0} \backslash\{k\}}^{\varepsilon}, \omega^{0}-p\left(R_{k}^{0}\right)\right)>p\left(R_{i}^{\varepsilon}\right) .
$$

By definition of the profile $R_{N^{0}}^{\varepsilon}$ however, $f_{i}\left(R_{N^{0}}^{\varepsilon}, \omega^{0}\right)=p\left(R_{i}^{\varepsilon}\right)$, a contradiction. Thus, by efficiency, $f_{k}\left(R_{N^{0}}^{\varepsilon}, \omega^{0}\right)>p\left(R_{k}^{0}\right)$. QED

Now fix an agent $k \in N^{0} \backslash\{i, j\}$ and consider a sequence $\left\{\varepsilon^{t}\right\}_{t=0}^{\infty} \longrightarrow 0$ such that for all $t \in \mathbb{N}, 0<\varepsilon^{t}<m_{i}^{s}\left(\{i, j\}, R_{N^{0} \backslash\{i, j, k\}}^{0}, \omega^{0}-p\left(R_{k}^{0}\right)\right)$. By our supposition, there exist two sequences $\left\{R_{i}^{\varepsilon^{t}}\right\}_{t=0}^{\infty}$ and $\left\{R_{j}^{\varepsilon^{t}}\right\}_{t=0}^{\infty}$ such that for all $t \in \mathbb{N}, p\left(R_{i}^{\varepsilon^{t}}\right)+p\left(R_{j}^{\varepsilon^{t}}\right)+\sum_{N^{0} \backslash\{i, j\}} p\left(R_{l}^{0}\right)<\omega^{0}$ and $f_{i}\left(R_{i}^{\varepsilon^{t}}, R_{j}^{\varepsilon^{t}}, R_{N^{0} \backslash\{i, j\}}^{0}, \omega^{0}\right)=p\left(R_{i}^{\varepsilon^{t}}\right)$. 
By the previous claim and by our assumption that $p\left(R_{N^{0} \backslash\{i, j\}}^{0}\right)>0$, for all $t \in \mathbb{N}$ we have

$$
f_{k}\left(R_{i}^{\varepsilon^{t}}, R_{j}^{\varepsilon^{t}}, R_{k}^{0}, R_{N^{0} \backslash\{i, j, k\}}^{0}, \omega^{0}\right)>p\left(R_{k}^{0}\right)>0 .
$$

Now let $R_{k}^{*} \in \mathcal{R}$ be such that $p\left(R_{k}^{*}\right)=0$. Then, by Lemma 3.2,

$$
f_{k}\left(R_{i}^{\varepsilon^{t}}, R_{j}^{\varepsilon^{t}}, R_{k}^{*}, R_{N^{0} \backslash\{i, j, k\}}^{0}, \omega^{0}\right)=f_{k}\left(R_{i}^{\varepsilon^{t}}, R_{j}^{\varepsilon^{t}}, R_{k}^{0}, R_{N^{0} \backslash\{i, j, k\}}^{0}, \omega^{0}\right)
$$

and thus, by consistency $f\left(R_{i}^{\varepsilon^{t}}, R_{j}^{\varepsilon^{t}}, R_{k}^{*}, R_{N^{0} \backslash\{i, j, k\}}^{0}, \omega^{0}\right)=f\left(R_{i}^{\varepsilon^{t}}, R_{j}^{\varepsilon^{t}}, R_{k}^{0}, R_{N^{0} \backslash\{i, j, k\}}^{0}, \omega^{0}\right) .{ }^{21}$

Now given a preference relation $R \in \mathcal{R}$, let $\bar{R} \in \mathcal{R}$ be the symmetric preference relation with the same peak, that is, let $p(\bar{R})=p(R)$ and for all $\alpha \in[0, p(\bar{R})],(p(\bar{R})-\alpha) \bar{I}(p(\bar{R})+\alpha)$. Then by Lemma 3.1,

$$
f_{i}\left(\bar{R}_{i}^{\varepsilon^{t}}, R_{j}^{\varepsilon^{t}}, R_{k}^{*}, R_{N^{0} \backslash\{i, j, k\}}^{0}, \omega^{0}\right)=f_{i}\left(R_{i}^{\varepsilon^{t}}, R_{j}^{\varepsilon^{t}}, R_{k}^{*}, R_{N^{0} \backslash\{i, j, k\}}^{0}, \omega^{0}\right)
$$

and by consistency,

$$
f\left(\bar{R}_{i}^{\varepsilon^{t}}, R_{j}^{\varepsilon^{t}}, R_{k}^{*}, R_{N^{0} \backslash\{i, j, k\}}^{0}, \omega^{0}\right)=f\left(R_{i}^{\varepsilon^{t}}, R_{j}^{\varepsilon^{t}}, R_{k}^{*}, R_{N^{0} \backslash\{i, j, k\}}^{0}, \omega^{0}\right) .
$$

Similarly, by Lemma 3.1 and consistency,

$$
f\left(\bar{R}_{i}^{\varepsilon^{t}}, \bar{R}_{j}^{\varepsilon^{t}}, R_{k}^{*}, R_{N^{0} \backslash\{i, j, k\}}^{0}, \omega^{0}\right)=f\left(\bar{R}_{i}^{\varepsilon^{t}}, R_{j}^{\varepsilon^{t}}, R_{k}^{*}, R_{N^{0} \backslash\{i, j, k\}}^{0}, \omega^{0}\right) .
$$

Overall, for each $t \in \mathbb{N}$

$$
f_{i}\left(\bar{R}_{i}^{\varepsilon^{t}}, \bar{R}_{j}^{\varepsilon^{t}}, R_{k}^{*}, R_{N^{0} \backslash\{i, j, k\}}^{0}, \omega^{0}\right)=p\left(\bar{R}_{i}^{\varepsilon^{t}}\right)
$$

and

$$
f_{k}\left(\bar{R}_{i}^{\varepsilon^{t}}, \bar{R}_{j}^{\varepsilon^{t}}, R_{k}^{*}, R_{N^{0} \backslash\{i, j, k\}}^{0}, \omega^{0}\right)>p\left(R_{k}^{0}\right)>0 .
$$

Let $R_{i}^{*} \in \mathcal{R}$ be such that $p\left(R_{i}^{*}\right)=0$. Since for all $t \in \mathbb{N}, p\left(\bar{R}_{i}^{\varepsilon^{t}}\right)<\varepsilon^{t}$ and since $\lim _{t \rightarrow \infty} \varepsilon^{t}=$ 0 , we have $\lim _{t \rightarrow \infty} p\left(\bar{R}_{i}^{\varepsilon^{t}}\right)=0$ and thus, $\lim _{t \rightarrow \infty} \bar{R}_{i}^{\varepsilon^{t}}=R_{i}^{*}$. Now note that $\left\{p\left(\bar{R}_{j}^{\varepsilon^{t}}\right)\right\}_{t=0}^{\infty}$ is a

\footnotetext{
${ }^{21}$ Though this is a typical application of consistency, let us clarify. Let $z=f_{k}\left(R_{i}^{\varepsilon^{t}}, R_{j}^{\varepsilon^{t}}, R_{k}^{*}, R_{N^{0} \backslash\{i, j, k\}}^{0}, \omega^{0}\right)$. Then by consistency, $f_{N^{0} \backslash\{k\}}\left(R_{i}^{\varepsilon^{t}}, R_{j}^{\varepsilon^{t}}, R_{k}^{*}, R_{N^{0} \backslash\{i, j, k\}}^{0}, \omega^{0}\right)=f_{N^{0} \backslash\{k\}}\left(R_{i}^{\varepsilon^{t}}, R_{j}^{\varepsilon^{t}}, R_{N^{0} \backslash\{i, j, k\}}^{0}, \omega^{0}-z\right)$. Also by consistency, $f_{N^{0} \backslash\{k\}}\left(R_{i}^{\varepsilon^{t}}, R_{j}^{\varepsilon^{t}}, R_{k}^{0}, R_{N^{0} \backslash\{i, j, k\}}^{0}, \omega^{0}\right)=f_{N^{0} \backslash\{k\}}\left(R_{i}^{\varepsilon^{t}}, R_{j}^{\varepsilon^{t}}, R_{N^{0} \backslash\{i, j, k\}}^{0}, \omega^{0}-z\right)$. This establishes the desired equality.
} 
sequence in the compact set $Z$. It therefore has a convergent subsequence $\left\{p\left(\bar{R}_{j}^{\varepsilon^{s(t)}}\right)\right\}_{t=0}^{\infty}$. Let $R_{j}^{*} \in \mathcal{R}$ be the symmetric preference relation such that $p\left(R_{j}^{*}\right)=\lim _{t \rightarrow \infty} p\left(\bar{R}_{j}^{\varepsilon^{s(t)}}\right)$. Then $\lim _{t \rightarrow \infty} \bar{R}_{j}^{\varepsilon^{s(t)}}=R_{j}^{*}$. Thus

$$
\lim _{t \rightarrow \infty}\left(\bar{R}_{i}^{\varepsilon^{s(t)}}, \bar{R}_{j}^{\varepsilon^{s(t)}}, R_{k}^{*}, R_{N^{0} \backslash\{i, j, k\}}^{0}, \omega^{0}\right)=\left(R_{i}^{*}, R_{j}^{*}, R_{k}^{*}, R_{N^{0} \backslash\{i, j, k\}}^{0}, \omega^{0}\right) .
$$

Therefore, by continuity

$$
f_{i}\left(R_{i}^{*}, R_{j}^{*}, R_{k}^{*}, R_{N^{0} \backslash\{i, j, k\}}^{0}, \omega^{0}\right)=p\left(R_{i}^{*}\right)=0
$$

and

$$
f_{k}\left(R_{i}^{*}, R_{j}^{*}, R_{k}^{*}, R_{N^{0} \backslash\{i, j, k\}}^{0}, \omega^{0}\right) \geq p\left(R_{k}^{0}\right)>0 .
$$

Let $\omega^{*}=f_{k}\left(R_{i}^{*}, R_{j}^{*}, R_{k}^{*}, R_{N^{0} \backslash\{i, j, k\}}^{0}, \omega^{0}\right)$. Thus by consistency,

$$
f\left(R_{i}^{*}, R_{k}^{*}, \omega^{*}\right)=\left(0, \omega^{*}\right) .
$$

Note that $p\left(R_{i}^{*}\right)=p\left(R_{k}^{*}\right)=0<\omega^{*}$. This, however, contradicts Lemma 3.3. 